\title{
Effects of Pinacidil on Refractoriness in Acutely Ischemic Tissue: Insights from Experiments and Simulations
}

\author{
B Trenor ${ }^{1}$, JM Ferrero (Jr) ${ }^{1}, \mathrm{~J} \mathrm{Saiz}^{1}$, A Ferrero $^{2}, \mathrm{~J}^{3}$ Chorro $^{2}$, \\ M Monserrat $^{1}, \mathrm{~V}_{\text {Hernández }}{ }^{3}, \mathrm{G}_{\text {Moltó }}{ }^{3}$ \\ ${ }^{1}$ Center for Research and Innovation in Bioengineering, Universidad Politecnica de Valencia, Spain \\ ${ }^{2}$ Servicio de Cardiologia, Hospital Clinico Universitario de Valencia, Spain \\ ${ }^{3}$ Grupo de Redes y Computación de Altas Prestaciones, Universidad Politecnica de Valencia, Spain
}

\begin{abstract}
A controversy exits regarding the arrhythmogenic effect of potassium channel openers, such as pinacidil, during acute myocardial ischemia. In order to throw some insight into this issue, the effect of pinacidil on the refractoriness of normal and acutely ischemic tissue has been experimentally and theoretically studied. Experiments with Langendorff perfused rabbit hearts were conducted, as well as computer simulations based on Luo-Rudy phase II model of the cardiac action potential. Our results show that pinacidil significantly reduces the effective refractory period (ERP) in normal tissue. However, in spite of strongly reducing QT interval, pinacidil does not have a significant effect on ERP in acutely ischemic tissue. In conclusion, during myocardial ischemia, despite action potential duration (APD) reduction provoked by pinacidil, ERP is not significantly modified by the drug due to the counteracting effect of $I_{K(A T P)}$ on depolarization currents
\end{abstract}

\section{Introduction}

Potassium channel openers, such as pinacidil, are known to be vasodilatory drugs with controversial effects on arrhythmogenic processes. Many experimental studies addressing the behavior of this drug suggest that pinacidil increases the potential for the development of ventricular fibrillation under different circumstances, such as myocardial ischemia or hypoxia [1-3]. On the contrary, other authors sustain the hypothesis of the dosedependent antiarrhythmic effect of pinacidil [4,5], especially in arhythmogenic episodes related to triggered activity.

Focussing the effect of pinacidil on arrhythmogenic processes related to reentrant activity and arising during acute myocardial ischemia, it is important to understand the electrophysiological changes that take place under such circumstances. Indeed, during myocardial ischemia metabolic impairment, potassium accumulation, acidosis and hypoxia provoke important changes in the electrical activity of the ischemic cells, creating inhomogeneities within the tissue and thus setting the stage for reentry. Special attention must be paid to the action potential duration (APD) reduction and refractoriness changes provoked by ATP-sensitive potassium channels (K(ATP) channels) activation. These effects concomittant with conduction velocity alteration are responsible, in part, for reentry generation and maintenance [6].

As pinacidil enhances ATP-sensitive potassium current $\left(\mathrm{I}_{\mathrm{K}(\mathrm{ATP})}\right)$ [7], it is relevant to study the effect of this drug on APD and effective refractory period (ERP) in order to evaluate possible actions on arrhythmogenic episodes related to myocardial ischemia. In normoxia, pinacidil significantly reduces ERP [8,9], however these changes under acute myocardial ischemia are yet to be addressed.

The goal of this study was to analyze experimentally and theoretically, by means of computer simulation, changes in APD and ERP under normal and acute ischemic circumstances in the presence of pinacidil.

\section{Methods}

\subsection{Experimental study}

Fourteen New Zealand rabbits were anesthetized with intravenous ketamine and then heparinized. The heart was immediately removed and immersed in cold $\left(4^{\circ} \mathrm{C}\right)$ Tyrode solution. After isolation, the aorta was connected to a Langendorff system for perfusion with Tyrode solution at a temperature of $37^{\circ} \mathrm{C}$. The heart was oxygenated with a mixture of $95 \% \mathrm{O}_{2}$ and $5 \% \mathrm{CO}_{2}$. The ventricles were electrically stimulated by means of a bipolar electrode using a GRASS S800 (Grass Instruments Inc.) programmable stimulator. A train of basic stimuli with a basic cycle length (BCL) of $250 \mathrm{~ms}$ with delivered prior to the test stimulus which was used to determine the ERP. Electrograms were recorded by a multi-electrode array of 256 electrodes $(1 \mathrm{~mm}$ interelectrode distance). In the pinacidil group, $10 \mu \mathrm{mol} / \mathrm{L}$ of pinacidil was administrated 
by dissolving rthe drug in the Tyrode solution. In the ischemia group, the circumflex artery was ligated and recordings were taken 5 minutes after artery ligation.

\subsection{Simulation study}

In order to simulate the electrical activity of ventricular cells we used a modified version of Luo-Rudy Phase II action potential (AP) model [10] [11], in which $\mathrm{I}_{\mathrm{K}(\mathrm{ATP})}$ current formulation [12] and the action of pinacidil on this current [13] were included.

We considered a 1D strand of 200 cells and propagation of action potential was studied. Firstly, normal physiological conditions were considered, and APD and ERP were measured in the absence and presence of $10 \mu \mathrm{mol} / \mathrm{L}$ of pinacidil. Secondly, physiological conditions were altered so as to reproduce an acute ischemic episode. Indeed, metabolical impairment was mimiqued by reducing intracellular ATP concentration from normoxic values $(6.8 \mathrm{mmol} / \mathrm{L})$ to 4.4 $\mathrm{mmol} / \mathrm{L}$ and increasing intracellular ADP concentration from typical normoxic values $(15 \mathrm{mmol} / \mathrm{L})$ to 150 $\mathrm{mmol} / \mathrm{L}$ [14]. Acidosis, which also arises during myocardial ischemia, is known to block partially sodium and calcium channels [15-17], and thus these currents were multiplied by 0.9 . Finally, extracellular potassium concentration was increased from normoxic values (5.4 $\mathrm{mmol} / \mathrm{L})$ to ischemic values $(11 \mathrm{mmol} / \mathrm{L})$ [14]. Under these circumstances, APD and ERP were also measured in the absence and presence of $10 \mu \mathrm{mol} / \mathrm{L}$ of pinacidil.

The protocol of stimulation is depicted in figure 1. A premature current pulse was applied at different coupling intervals (CI) after the basic stimulus given at $\mathrm{t}=50 \mathrm{~ms}$. Their amplitudes were 1.5 times diastolic threshold and the duration was $2 \mathrm{~ms}$.

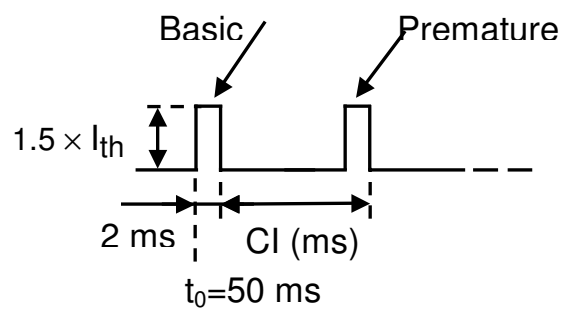

Figure 1. Stimulation protocol.

Both current pulses were applied to one edge of the strand. The ERP was measured as the shortest CI leading to AP propagation. APD was measured at $90 \%$ of repolarization.

\section{Results}

In fist place, the ERP was experimentally measured under different situations. The results were compared and are depicted in Figure 2. In analyzing the effects of ischemia on the ERP, we compared control ERPs (first and third bars) with ischemic ERPs (second and fourth bars), in the absence (first two bars) and presence of 10 $\mu \mathrm{mol} / \mathrm{L}$ pinacidil (second two bars). ERP values were normalized to the control value in the absence of the drug. A significative decrease of ERP was observed in the absence of the drug, however the difference was nonsigniticative (NS) in the presence of pinacidil.

As for the effects of pinacidil on ERP, normal cells recovered excitability before in the presence of pinacidil (bar six) than in its absence (bar five), and the difference was significative. On the contrary, this difference was non-significative (NS) under ischemic conditions (two last bars). This last observation determined the second part of our study.

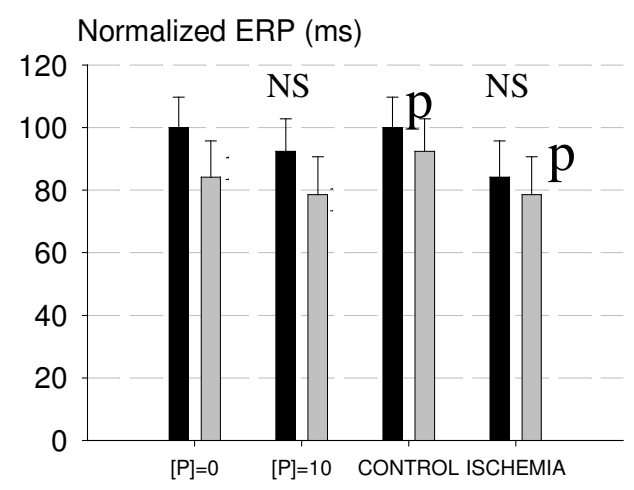

Figure 2. Experimental measured and normalized ERPs. C stands for control, i for ischemia and $\mathrm{P}$ for pinacidil.

In second place, computer simulations were conducted in order to throw some insight into the mechanism of action of pinacidil. The above described experiments were simulated and similar results were obtained.

Pinacidil strongly reduced APD under ischemic conditions, as shown in Figure 3. A less important decrease was also observed in normoxia (results not shown). As expected, also ischemia shortened APD in the presence, as well as in the absence of the drug.

Pinacidil and ischemia also reduced ERP as shown in table 1 .

Table 1. Simulated ERP in milliseconds

\begin{tabular}{|l|l|l|}
\hline & Control & Ischemia \\
\hline$[\mathrm{P}]=0$ & 180 & 147 \\
\hline$[\mathrm{P}]=10 \mu \mathrm{mol} / \mathrm{L}$ & 161 & 144 \\
\hline
\end{tabular}

From the obtained results, it can be deduced that the reduction in ERP provoked by pinacidil was less 
important under ischemic conditions, results also observed experimentally.

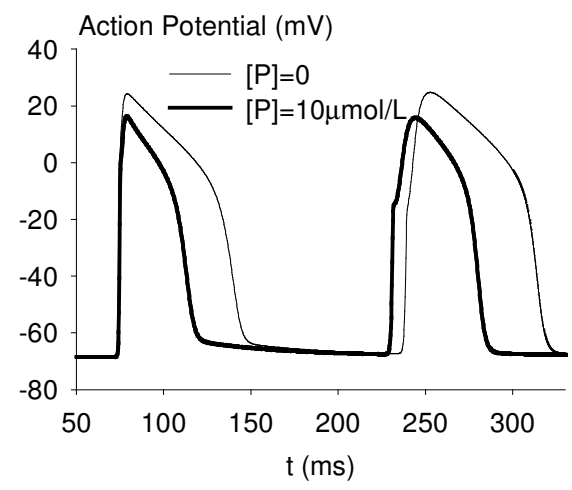

Figure 3. Basic and first propagated premature action potentials in the absence and presence of $10 \mu \mathrm{mol} / \mathrm{L}$ pinacidil, under ischemic conditions.

Our analysis was then foccussed on trying to understand this effect.

As depicted in Figure 3, allthough APD was strongly reduced by pinacidil under ischemia (from 72 milliseconds in the absence of the drug to 45 milliseconds in the presence of pinacidil), reduction of ERP was not so remarkable. Indeed, in the absence of the drug, a CI of 147 milliseconds led to AP propagation, whereas under the effects of pinacidil a CI of 144 milliseconds led to AP propagation. Postrepolarization refractoriness was then 24 milliseconds longer in the second case.

Ionic currents were also measured under these ischemic conditions in order to analyze the effect of the drug. Figure 4 shows the time course of $\mathrm{I}_{\mathrm{K}(\mathrm{ATP})}$ outward current and inward sodium current $\left(\mathrm{I}_{\mathrm{Na}}\right)$ in both cases. The enhanced activity of $\mathrm{K}$ (ATP) channels in ischemia is further increased by pinacidil. And finally, the inward sodium current must reach a higher level in the presence of the drug to allow AP propagation.

\section{Discussion and conclusions}

Several experimental studies have pointed out the dose dependent effects of pinacidil on arrhythmias $[1,3,4,18,19]$. Particularly, reentrant arrhythmias arising during the acute phase of myocardial ischemia are subjected to the effect of this drug. Under such circumstances, much attention should be paid to electophysiological alterations related to refractoriness and APD, which are involved in reentrant circuit generation and maintenance [6].

This study focussed on APD and ERP alterations caused by pinacidil under the acute phase of myocardial ischemia or normoxia. The broad experimental literature has generated extensive data on APD and ERP shortening

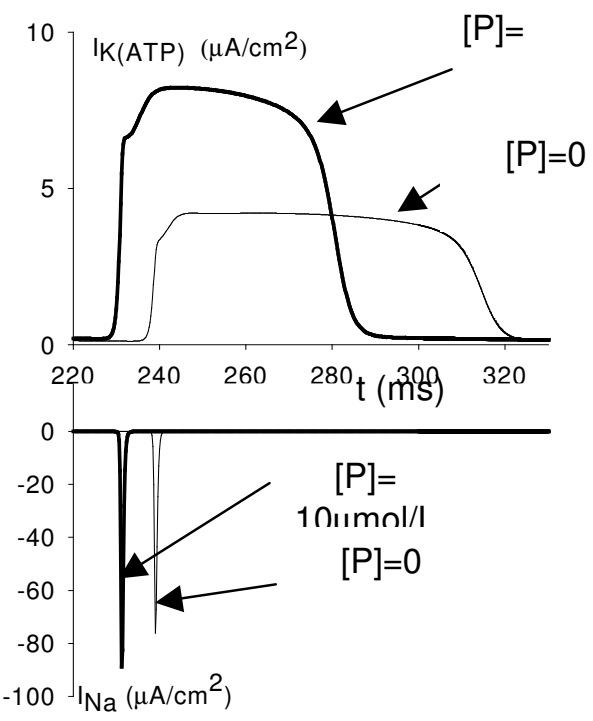

Figure 4. $\mathrm{I}_{\mathrm{KATP}}$ and $\mathrm{I}_{\mathrm{Na}}$ currents during ischemia in the absence and in the presence of $10 \mu \mathrm{mol} / \mathrm{L}$ pinacidil, after the premature stimulus.

by pinacidil under normal physiological conditions $[8,20]$. Our experimental and simulation results are in accordance with these observations.

If we consider the effects of the drug on ERP under pathological situations, such as the acute phase of ischemia, little experimental or theoretical results have been documented. The present study suggests that the difference between ERP reduction caused by pinacidil under normoxia and under ischemia is non-significative. Our experimental conditions were mimicked by computer simulations in order to investigate ionic mechanisms associated with pinacidil effects on refractoriness. As related in the previous section, during ischemia, postrepolarization refractoriness was longer in the presence of the drug. Indeed, in the presence of the drug APD shortening was greater than ERP shortening. A shorter APD in the presence of pinacidil allows a sooner recuperation of refractoriness, so as to expect a significantly shorter ERP. However, an enhanced $\mathrm{I}_{\mathrm{K}(\mathrm{ATP})}$ current under the effects of pinacidil (see Figure 4), counteracts depolarizing sodium current, and thus $\mathrm{I}_{\mathrm{Na}}$ must reach higher values (see Figure 4) to assure action potential upstroke and propagation. Consequently, CI must be longer enough to assure sodium channels availability, so that ERP is not significantly reduced.

The above described effects exerted by pinacidil on ERP can have important consequences on arrhythmogenesis. Moreover its intricate mechanism of action colud respond for the existent controversy in the experimental litterature, concerning the proarrhythmic 
action of this drug. Two opposite effects in terms of refractoriness must be taken into account. On the one hand, the activation of $\mathrm{K}_{\text {(ATP) }}$ channels provoked by pinacidil, reduces significantly APD, so that the recovery of excitability starts before reducing ERP. On the other hand, the enhanced activity of $\mathrm{I}_{\mathrm{K} \text { (ATP) }}$ counteracts depolarization currents so that postrepolarization refractoriness can be increased. The administered dose of the drug is then determinant to evaluate its net effect on refractoriness and thus on arrhythmogenic activity related to reentrant circuits. Also experimental and physiological conditions are extremely important in determining the action exerted by the drug.

\section{Acknowledgements}

This work was partially supported by (1) the Plan Nacional de Investigación Científica, Desarrollo e Innovación Tecnológica del Ministerio de Ciencia y Tecnología of Spain (TIC 2001-2686) and (2) the Programa Incentivo a la Investigación de la Universidad Politecnica de Valencia (CAMAV Project 20020418).

\section{References}

[1] Wolleben CD, Sanguinetti MC, Siegl PK. Influence of ATP-sensitive potassium channel modulators on ischemiainduced fibrillation in isolated rat hearts. J. Mol. Cell Cardiol. 1989; 21: 783-788.

[2] Chi L, Uprichard AC, Lucchesi BR. Profibrillatory actions of pinacidil in a conscious canine model of sudden coronary death. J. Cardiovasc. Pharmacol. 1990; 15: 452464.

[3] D'Alonzo AJ, Zhu JL, Darbenzio RB, Dorso CR, Grover GJ. Proarrhythmic effects of pinacidil are partially mediated through enhancement of catecholamine release in isolated perfused guinea-pig hearts. J. Mol. Cell Cardiol. 1998; 30: 415-423.

[4] D'Alonzo AJ, Darbenzio RB, Hess TA, Sewter JC, Sleph PG, Grover GJ. Effect of potassium on the action of the KATP modulators cromakalim, pinacidil, or glibenclamide on arrhythmias in isolated perfused rat heart subjected to regional ischaemia. Cardiovasc. Res. 1994; 28: 881-887.

[5] Carlsson L, Abrahamsson C, Drews L, Duker G. Antiarrhythmic effects of potassium channel openers in rhythm abnormalities related to delayed repolarization. Circulation 1992; 85: 1491-1500.

[6] Nassif G, Dillon SM, Rayhill S, Wit AL. Reentrant circuits and the effects of heptanol in a rabbit model of infarction with a uniform anisotropic epicardial border zone. J. Cardiovasc. Electrophysiol. 1993; 4: 112-133.

[7] Fan Z, Nakayama K, Hiraoka M. Multiple actions of pinacidil on adenosine triphosphate-sensitive potassium channels in guinea-pig ventricular myocytes. J. Physiol 1990; 430: 273-295.

[8] Sanguinetti MC, Scott AL, Zingaro GJ, Siegl PK. BRL 34915 (cromakalim) activates ATP-sensitive K+ current in cardiac muscle. Proc. Natl. Acad. Sci. U. S. A 1988; 85: 8360-8364.
[9] Friedrichs GS, Chi L, Black SC, Manley PJ, Lucchesi BR. Antiarrhythmic agent, MS-551, protects against pinacidil + hypoxia- induced ventricular fibrillation in Langendorffperfused rabbit isolated heart. J. Cardiovasc. Pharmacol. 1994; 23: 120-126.

[10] Luo CH, Rudy Y. A dynamic model of the cardiac ventricular action potential. I. Simulations of ionic currents and concentration changes. Circ. Res. 1994; 74: 1071-96.

[11] Zeng J, Laurita KR, Rosenbaum DS, Rudy Y. Two components of the delayed rectifier $\mathrm{K}+$ current in ventricular myocytes of the guinea pig type. Theoretical formulation and their role in repolarization. Circ. Res. 1995; 77: 140-152.

[12] Ferrero JM, Jr., Saiz J, Ferrero JM, Thakor NV. Simulation of action potentials from metabolically impaired cardiac myocytes. Role of ATP-sensitive K+ current. Circ. Res. 1996; 79: 208-221.

[13] Trenor B, Ferrero JM, Jr., Rodriguez B, Saiz J, Ferrero JM, Thakor N. Simulation Study of the Effect of Pinacidil on ATP-Sensitive Potassium Current and Action Potential Duration in Myocardial Tissue. Computers in Cardiology 1998; 25:581-584.

[14] Weiss JN, Venkatesh N, Lamp ST. ATP-sensitive K+ channels and cellular $\mathrm{K}+$ loss in hypoxic and ischaemic mammalian ventricle. J. Physiol 1992; 447: 649-673.

[15] Watson CL, Gold MR. Effect of intracellular and extracellular acidosis on sodium current in ventricular myocytes. Am. J. Physiol 1995; 268: H1749-H1756.

[16] Shaw RM, Rudy Y. Electrophysiologic effects of acute myocardial ischemia. A mechanistic investigation of action potential conduction and conduction failure. Circ. Res. 1997; 80: 124-138.

[17] Kagiyama Y, Hill JL, Gettes LS. Interaction of acidosis and increased extracellular potassium on action potential characteristics and conduction in guinea pig ventricular muscle. Circ. Res. 1982; 51: 614-623.

[18] Padrini R, Bova S, Cargnelli G, Piovan D, Ferrari M. Effects of pinacidil on guinea-pig isolated perfused heart with particular reference to the proarrhythmic effect. Br. J. Pharmacol. 1992; 105: 715-719.

[19] Lepran I, Baczko I, Varro A, Papp JG. ATP-sensitive potassium channel modulators: both pinacidil and glibenclamide produce antiarrhythmic activity during acute myocardial infarction in conscious rats. J. Pharmacol. Exp. Ther. 1996; 277: 1215-1220.

[20] Friedrichs GS, Chi L, Black SC, Manley PJ, Lucchesi BR. Antiarrhythmic agent, MS-551, protects against pinacidil + hypoxia-induced ventricular fibrillation in Langendorffperfused rabbit isolated heart. J. Cardiovasc. Pharmacol. 1994; 23: 120-126.

Address for correspondence.

Beatriz Trenor.

Universidad Politecnica de Valencia

Camino de Vera s/n Valencia 46022. Spain.

btrenor@eln.upv.es 